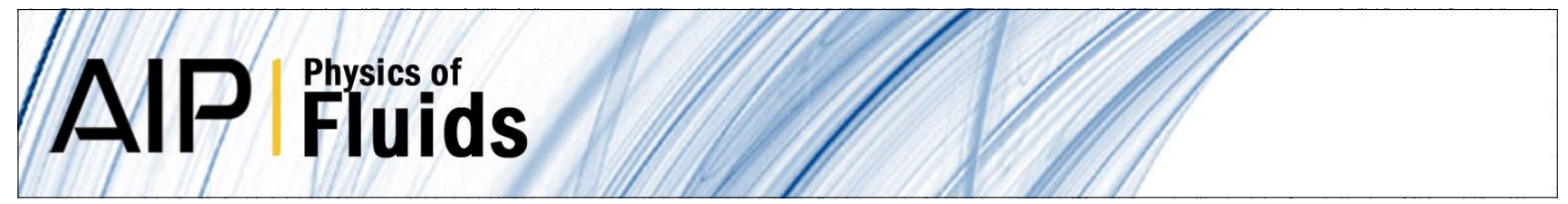

\title{
Investigation of the steady engulfment regime in a three-dimensional T- mixer
}

Andrea Fani, Simone Camarri, and Maria Vittoria Salvetti

Citation: Phys. Fluids 25, 064102 (2013); doi: 10.1063/1.4809591

View online: http://dx.doi.org/10.1063/1.4809591

View Table of Contents: http://pof.aip.org/resource/1/PHFLE6/v25/i6

Published by the American Institute of Physics.

\section{Additional information on Phys. Fluids}

Journal Homepage: http://pof.aip.org/

Journal Information: http://pof.aip.org/about/about_the_journal

Top downloads: http://pof.aip.org/features/most_downloaded

Information for Authors: http://pof.aip.org/authors

\section{ADVERTISEMENT}

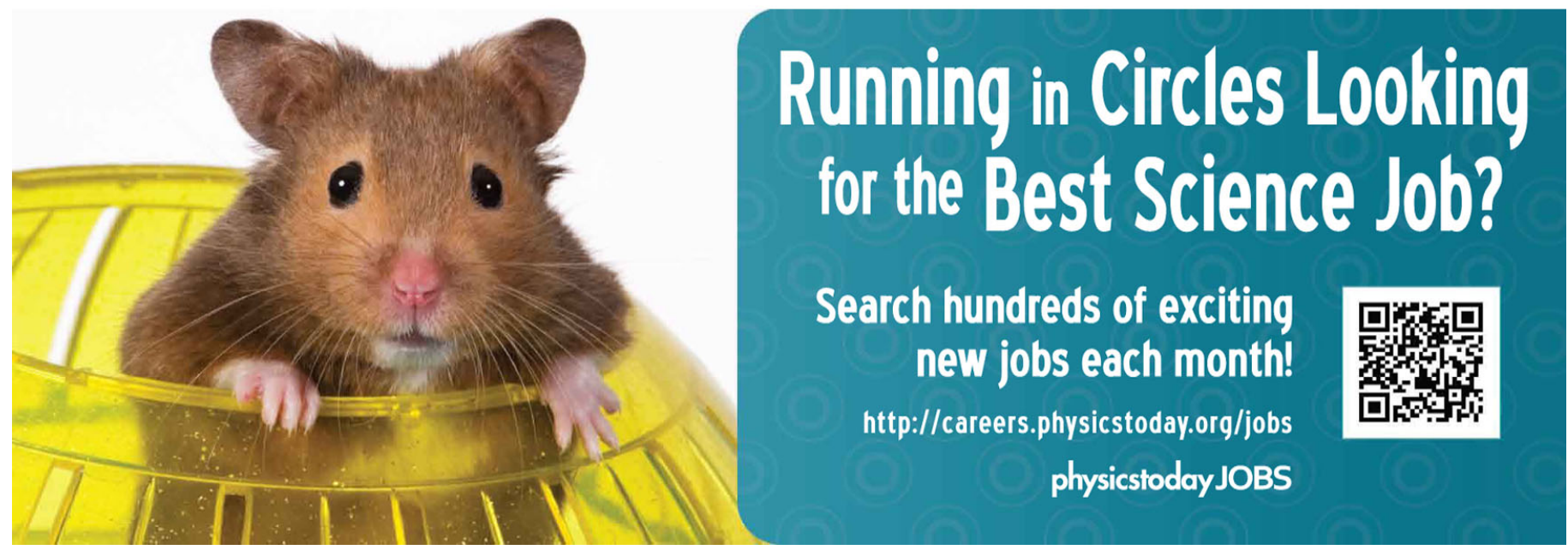




\title{
Investigation of the steady engulfment regime in a three-dimensional T-mixer
}

\author{
Andrea Fani, Simone Camarri, ${ }^{\text {a) }}$ and Maria Vittoria Salvetti \\ Dipartimento di Ingegneria Civile ed Industriale, Università di Pisa, via G. Caruso 8, \\ 56122 Pisa, Italy
}

(Received 13 March 2013; accepted 22 May 2013; published online 6 June 2013)

\begin{abstract}
The steady engulfment regime in a fully three-dimensional micro T-mixer is investigated. This regime is of significant interest for applications since it implies high mixing between the flow streams entering the device. Direct numerical simulations are first used to characterize this regime. In particular, the main vortical structures typical of the engulfment regime and their effects on mixing are investigated. Threedimensional linear stability analysis is successively applied to the characterization of the instability leading to the engulfment regime. The critical Reynolds number and the global unstable mode are first computed for a configuration characterized by fully-developed inlet velocity conditions. The sensitivity of this instability to a generic modification of the base flow is then investigated, thanks to the computation of the mode adjoint to the direct unstable one. Finally, this kind of analysis is specialized to investigate the effect of a perturbation of the velocity distribution at the inlet of the T-mixer. Sensitivity analysis shows that non-fully developed inlet velocity conditions lead to an increase of the critical Reynolds number. More generally, the sensitivity maps can be used for the design of control strategies aimed at promoting or inhibiting the engulfment. An example is provided for a control based on blowing/suction through the mixer walls. (C2013 AIP Publishing LLC. [http://dx.doi.org/10.1063/1.4809591]
\end{abstract}

\section{INTRODUCTION}

Micro-mixers are important devices in microfluidics, and for this reason they are widely investigated in the literature, with a particular attention to passive micro-mixers (see, e.g., Kumar et al. ${ }^{1}$ ), which are able to promote mixing without any external energy supply. Among the possible shapes of micro-mixers, the micro T-mixers are very common, and they are also often used simply as junction elements in complex micro-systems. When used as mixers, the flow enters from the two opposite pipes (inflow pipes) and the mixture outflows from the third pipe (outflow pipe or mixing channel). Although a plane mixer may be an interesting configuration for detailed theoretical analysis, the attention in the literature is mainly focused on 3D configurations with rectangular sections of the pipes for their practical interest. For this kind of mixers, three different working regimes have been identified in experiments and simulations available in the literature, depending on the flow Reynolds number (see, e.g., Engler et al., ${ }^{2}$ Bothe $e t$ al. ${ }^{3}$ and Hoffmann $e t$ al. ${ }^{4}$ ). At very low Reynolds numbers, the two streams coming from the inlet pipes remain completely segregated even in the outflow pipe (stratified flow) and mixing is only due to diffusion. As the Reynolds number is increased, a second regime is observed (vortex regime), in which a secondary flow, organized in a double pair of counterrotating vortices, is present in the outflow pipe. This flow preserves the two reflection symmetries of the device geometry. In this regime, mixing is only slightly increased because of the augmented contact surface between the two streams, which remain however segregated. For larger Reynolds numbers, an organized and stationary pattern of vortical structures is observed (engulfment regime),

\footnotetext{
a)s.camarri@ing.unipi.it
} 
which breaks the flow symmetries and makes fluid elements of one stream reach the opposite side of the mixing channel. This clearly leads to a significantly larger mixing than in the previous regimes. If the Reynolds number is further increased, the flow may become unsteady (see, e.g., Bothe et al., ${ }^{3}$ Dreher et al.,$^{5}$ and Galletti $\left.e t a l .{ }^{6}\right)$. For instance, for square inlet pipes and an outflow pipe width $\left(W_{o}\right)$ to height $(H)$ ratio $W_{o} / H=2$ and for fully developed inlet velocity conditions, Engler $e t a l{ }^{2}$ show that the flow is in stratified regime approximately for $R e<50$ (based on the outflow hydraulic diameter and mean velocity), in vortex regime for $50<R e<150$, engulfment starts at $R e \simeq 150$ and the flow is periodic for $240<R e<400$. Clearly, the critical values of the Reynolds number corresponding to the onset of the different regimes may vary with geometrical and flow parameters, such as the shape of the pipe cross sections or the inlet flow conditions.

Among the identified flow regimes, steady and periodic engulfment are the most interesting for applications since they lead to efficient mixing within the device. Many works in the literature are dedicated to characterize, in particular, the steady engulfment regime and to investigate the flow conditions for its onset. Bothe et al. ${ }^{3}$ investigate mixing properties in the engulfment regime through numerical simulations and provide validation against experimental data. An experimental investigation is documented in Hoffmann et al. ${ }^{4}$ In Soleymani et al. ${ }^{7}$ an empirical equation is proposed to determine the critical Reynolds number for the engulfment as a function of the channel geometry, by extrapolating a set of numerical simulations. More recently, Cherlo et al. ${ }^{8}$ investigated, both numerically and experimentally, the effect of channel depth on the onset of engulfment, while Yousefi et al. ${ }^{9}$ provided the pressure drop and the length of mixing zone for a wide range of T-mixer dimensions and flow Reynolds numbers, in the vortex and engulfment regimes. The effect of a variation of the incoming velocity distribution on the engulfment regime in a T-mixer was studied numerically in Galletti et al. ${ }^{6}$ it is shown that this has a significant impact on both the onset and the characteristics of engulfment. Orsi et al ${ }^{10}$ numerically investigated the engulfment regime in a T-shaped micromixer, comparing the cases where the two inlet fluids consist of both water, and water and ethanol.

In the present paper, the steady engulfment regime is studied and characterized by means of Direct Numerical Simulation (DNS) and, for the first time in the literature to the authors' knowledge, by means of global linear stability and sensitivity analysis. Previous numerical and experimental investigations of this regime mainly focus on the mixing efficiency and associated pressure drop. We focus herein on the identification of the vortical structures characteristics of the different flow regimes and, in particular, on their modifications when passing from the vortex to the engulfment regime. The role of these vortices on the mixing capabilities of the device is highlighted. In synergy with DNS, for the first time to the authors' knowledge, engulfment has been studied also by means of global stability analysis, which allows an accurate estimation of the critical conditions for the onset of the related instability and the identification of the associated unstable mode (see, e.g., the review by Theofilis ${ }^{11}$ ). Furthermore, the sensitivity of this instability to a local perturbation of the flow can be systematically obtained following the methods proposed in Bottaro et al., ${ }^{12}$ Giannetti and Luchini, ${ }^{13}$ and Marquet et al.,${ }^{14}$ which rely on adjoint methods. Besides a further characterization of the identified instability, this analysis also provides quantitative information on possible control strategies, which in the present case are mainly oriented to anticipate the instability instead of suppressing it. In particular, following Galletti et al., ${ }^{6}$ we use these tools to systematically characterize the sensitivity of the onset of engulfment with respect to a perturbation of the inflow velocity distribution. The same analysis also provides maps useful for the design of different control strategies. As an example, we quantify here the sensitivity of the engulfment instability with respect to the introduction of jets on the mixer walls. The work documented here can be straightforwardly generalized to a wide range of control mechanisms, including time-dependent pulsations of the inflow profile. It is important to underline that the case at issue is a fully $3 \mathrm{D}$ flow, i.e., without homogeneous directions, and very few examples of fully 3D global stability and sensitivity analysis exist in the literature, due to the associated computational complexity (see, e.g., Bagheri et al. ${ }^{15}$ ). Thus, the stability and sensitivity analyses of the present case are also challenging from a technical viewpoint. To this purpose, a set of numerical tools has been developed using the spectral-element code NEK5000, ${ }^{16}$ and they have been validated for a plane case against another set of tools that has been assessed in previous works (see Fani et al. ${ }^{17}$ ). 


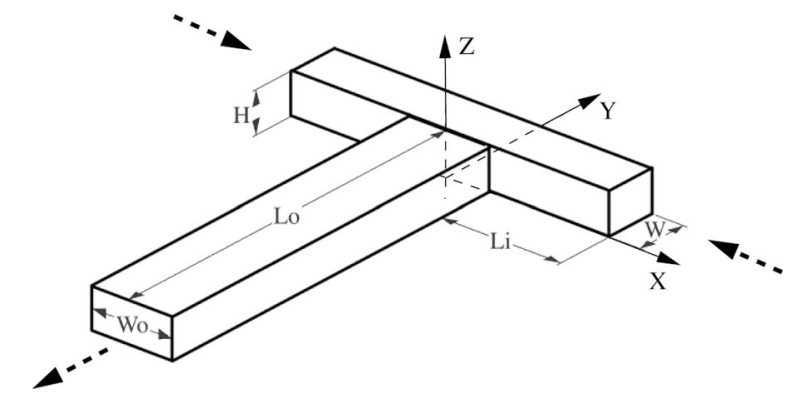

FIG. 1. Flow configuration and frame of reference; dashed lines indicate the flow direction.

\section{PROBLEM DESCRIPTION AND METHODOLOGY}

We consider here the incompressible flow of a Newtonian fluid inside a T-mixer, made by two inflow and one outflow pipes, all with rectangular cross sections. All quantities are normalized using as reference length and velocity the hydraulic diameter of the outflowing pipe and the bulk velocity of the inlet flow. With reference to Fig. 1 (not in scale), the height of the mixer is $H \simeq 0.83$, the width of the incoming pipes is $W=0.625$ and the width of the outcoming pipe is $W_{o}=2 W=1.25$, the aspect ratio of the incoming and outcoming cross sections being equal to $W / H=0.75$ and $W_{o} / H=1.50$, respectively. In the present simulations, the lengths of the inflow and outflow pipes are $L_{i}=6.875$ and $L_{o}=12.5$, and the incoming flow is assumed to have a fully developed (unidirectional) laminar profile for the considered rectangular cross-section. ${ }^{3}$ The same flow studied here has been investigated in Galletti et al., ${ }^{6}$ where it is shown that the considered lengths of the pipes are sufficient for a proper prediction of the steady engulfment instability.

The fluid motion is described by the three-dimensional unsteady incompressible Navier-Stokes equations

$$
\begin{aligned}
& \frac{\partial \mathbf{U}}{\partial t}+\mathbf{U} \cdot \nabla \mathbf{U}+\nabla P-\frac{1}{R e} \nabla^{2} \mathbf{U}=\mathbf{0}, \\
& \nabla \cdot \mathbf{U}=0,
\end{aligned}
$$

where $\mathbf{U}$ is the velocity vector with components $\mathbf{U}=(U, V, W)$ and $P$ is the reduced pressure. No-slip boundary conditions are imposed at the mixer walls, while at the inlet a unidirectional fully developed velocity profile is assumed. Finally, free outflow conditions of the following type are used: $\partial_{y} U=0, P-R e^{-1} \partial_{y} V=0 \partial_{y} W=0$.

The instability onset is studied using linear theory and normal-mode analysis. The flow variables are decomposed in the base flow $\left(\mathbf{U}_{b}, P_{b}\right)$, steady solution of Eqs. (1), and in an unsteady perturbation $(\mathbf{u}, p)$. The perturbation is searched in the form of normal modes $\mathbf{q}(x, y, z, t)=(\mathbf{u}, p)(x, y, z, t)$ $=(\hat{\mathbf{u}}, \hat{p})(x, y, z) \exp (\sigma t)$, where $\sigma$ is a generally complex eigenvalue associated with the mode $(\hat{\mathbf{u}}, \hat{p})$. Introducing the flow decomposition and the normal-mode form into Eqs. (1) and linearizing with respect to the disturbance, we obtain the following equations governing the dynamics of the perturbation:

$$
\begin{aligned}
& \sigma \hat{\mathbf{u}}+\hat{\mathbf{u}} \cdot \nabla \mathbf{U}_{b}+\mathbf{U}_{\mathbf{b}} \cdot \nabla \hat{\mathbf{u}}+\nabla \hat{p}-\frac{1}{R e} \nabla^{2} \hat{\mathbf{u}}=\mathbf{0}, \\
& \nabla \cdot \hat{\mathbf{u}}=0 .
\end{aligned}
$$

The boundary conditions associated with Eqs. (2) are: $\hat{\mathbf{u}}=0$ at the inlet surfaces and on the walls, and free outflow conditions at the outlet. Equations (2) along with the boundary conditions are an eigenvalue problem. The flow is linearly stable when all the eigenvalues, $\sigma=\lambda+1 \omega$, are characterized by $\lambda<0$.

In the following, we concisely report the main steps needed to compute the sensitivity of the considered instability to a perturbation of the boundary conditions. In Giannetti and Luchini ${ }^{13}$ it is 
shown that the core of the instability associated with the global mode $(\hat{\mathbf{u}}, \hat{p}, \sigma)$ can be identified by inspecting the field $\|\hat{\mathbf{u}}\|\left\|\mathbf{u}^{+}\right\|$, where $\|\cdot\|$ denotes the $L_{2}$ norm. The vector field $\hat{\mathbf{u}}^{+}$is the velocity field of the mode adjoint to $(\hat{\mathbf{u}}, \hat{p}, \sigma)$, solution of the following eigenvalue problem:

$$
\begin{aligned}
& \sigma^{*} \hat{\mathbf{u}}^{+}+\nabla \mathbf{U}_{b} \cdot \hat{\mathbf{u}}^{+}-\mathbf{U}_{\mathbf{b}} \cdot \nabla \hat{\mathbf{u}}^{+}+\nabla \hat{p}^{+}-\frac{1}{R e} \nabla^{2} \hat{\mathbf{u}}^{+}=\mathbf{0}, \\
& \nabla \cdot \hat{\mathbf{u}}^{+}=0 .
\end{aligned}
$$

The boundary conditions associated with problem (3b) are: $\hat{\mathbf{u}}^{+}=0$ at the inlet surfaces and on the lateral walls, and $p^{+} \mathbf{n}-R e^{-1}\left(\mathbf{n} \cdot \nabla \hat{\mathbf{u}}^{+}\right)=\left(\mathbf{U}_{\mathbf{b}} \cdot \mathbf{n}\right) \hat{\mathbf{u}}^{+}$at the outlet (see Ref. 14). In the present work, we use the following condition to normalize the adjoint velocity field:

$$
\left(\hat{\mathbf{u}}^{+}, \hat{\mathbf{u}}\right)=1
$$

where $(a, b)=\int_{\Omega}\left(a^{*} \cdot b\right) d \Omega$ is the scalar product between the complex vectors $a$ and $b$ on the flow domain $\Omega$ and the asterisk denotes the conjugate of a complex quantity.

Bottaro et al. ${ }^{12}$ and Marquet et al. ${ }^{14}$ studied the modification $\delta \sigma$ of the eigenvalue caused by an arbitrary variation $\delta \mathbf{U}_{b}$ of the base flow. The eigenvalue drift can be written as follows:

$$
\delta \sigma=\frac{\left(M^{+}, \delta \mathbf{U}_{b}\right)}{\left(\hat{\mathbf{u}}^{+}, \hat{\mathbf{u}}\right)}
$$

where $M^{+}$is

$$
M^{+}=\hat{\mathbf{u}}^{*} \cdot \nabla \hat{\mathbf{u}}^{+}-\nabla \hat{\mathbf{u}}^{*} \cdot \hat{\mathbf{u}}^{+} .
$$

In the present work, we focus on the effect of a perturbation of the velocity distribution $\left(\delta \mathbf{U}_{\mathbf{i}}\right)$ at the inlet surfaces, which is a boundary condition for the problem and, in turn, induces a base flow modification $\delta \mathbf{U}_{b}$. The aim is to study the sensitivity of the engulfment with respect to the velocity distribution of the flow entering the mixer. The effect of the inlet perturbation on the instability could be quantified through Eq. (5), but this implies to compute the specific base-flow modifications $\delta \mathbf{U}_{b}$ for each perturbation of the inlet velocity. Following the approach of Marquet $e t$ al., ${ }^{14}$ the eigenvalue drift caused by a generic perturbation $\delta \mathbf{U}_{\mathbf{i}}$ of the inlet boundary conditions can be written as

$$
\delta \sigma=\frac{\left(P_{b}^{+} \mathbf{n}+R e^{-1} \mathbf{n}^{T} \cdot \nabla \mathbf{U}_{\mathbf{b}}^{+}, \delta \mathbf{U}_{\mathbf{i}}\right)_{\Gamma_{i}}}{\left(\hat{\mathbf{u}}^{+}, \hat{\mathbf{u}}\right)}
$$

where the subscript $\Gamma_{i}$ indicates a scalar product calculated on the inlet boundary of the domain, i.e., $(a, b)_{\Gamma_{i}}=\int_{\Gamma_{i}}\left(a^{*} \cdot b\right) d l$ (see also Ref. 18). In Eq. (7), $\mathbf{U}_{\mathbf{b}}{ }^{+}$and $P_{b}^{+}$are, respectively, the adjoint base flow velocity and the adjoint pressure, solution of the following problem:

$$
\begin{aligned}
& \nabla \mathbf{U}_{\mathbf{b}} \cdot \mathbf{U}_{b}^{+}-\mathbf{U}_{\mathbf{b}} \cdot \nabla \mathbf{U}_{\mathbf{b}}^{+}+\nabla P_{b}^{+}-\frac{1}{R e} \nabla^{2} \mathbf{U}_{\mathbf{b}}^{+}=M^{+}, \\
& \nabla \cdot \mathbf{U}_{\mathbf{b}}^{+}=0,
\end{aligned}
$$

along with homogeneous boundary conditions at the inlet surfaces and on the solid walls and the following condition on the outflow boundary: $P^{+} \mathbf{n}-R e^{-1}\left(\mathbf{n} \cdot \nabla \hat{\mathbf{U}}_{\mathbf{b}}^{+}\right)=-\left(\mathbf{U}_{\mathbf{b}} \cdot \mathbf{n}\right) \mathbf{U}_{\mathbf{b}}{ }^{+}$ $+\left(\hat{\mathbf{u}}^{*} \cdot \mathbf{n}\right) \hat{\mathbf{u}}^{+}$.

As concerns the numerical solution of the equations concisely recalled above, NEK5000 has been used, which is an open-source massively parallel spectral element code. ${ }^{16}$ The velocity space is spanned by Nth-order Lagrange polynomial interpolants, based on tensor-product arrays of GaussLobatto-Legendre (GLL) quadrature points in each hexahedral element, while the polynomial order for pressure is $N-2$. Time discretization uses explicit backward-differentiation for convective terms, and an implicit scheme for viscous terms. A third order scheme (BDF3) is used for the nonlinear Navier-Stokes equations, while the linearized equations are integrated in time by means of a second order scheme (BDF2). All the eigenvalue problems involved in the stability and sensitivity analysis are solved by a power method, using the linearized/adjoint version of NEK5000 as a time-stepper. 
For the validation of the numerical method described above, a plane T-mixer has also been considered, with the same geometry and dimensions of the 3D one investigated here, thus corresponding to the case of $H \rightarrow \infty$. For the plane case, the stability and sensitivity analyses have also been carried out through a set of tools that have been extensively validated in the past. ${ }^{17}$ In particular, in the 2D case the steady Navier-Stokes equations and the linearized equations for the perturbation evolution are discretized in space by a finite-element method, using P2-P1 Taylor-Hood elements. The meshes as well as the discrete matrices resulting from Galerkin finite-element method are generated with the software FreeFem++ (http://www.freefem.org by S. Auliac, F. Hecht, A. Le Hyaric, J. Morice, K. Ohtsuka, O. Pironneau). The steady nonlinear base-flow equations are solved via a Newton-Raphson iterative method. The generalized eigenvalue problems of the stability and sensitivity analyses are solved using a power method along with a shift-invert strategy, implemented with the library PETSc (Portable Extensible Toolkit for Scientific Computation). ${ }^{19}$ Details on the numerical method as well as of its validation can be found in Fani et al. ${ }^{17}$

\section{VALIDATION}

In this section the numerical tools for stability and sensitivity analysis built in the framework of NEK5000 are validated for a plane T-mixer against the results obtained by the finite-element codes $^{17}$ developed with FreeFem ++ . The numerics, as well as the dimensions of the plane mixer, are concisely described in Sec. II. Concerning the numerical discretization in NEK5000, a structured multi-block grid is used; the width of the pipes $\left(W_{o}\right.$ and $W$ ) is discretized by elements of size equal to 0.078 while the size of the elements along the axis of the pipes varies between 0.07 and 0.49 . Polynomials of order $N=9$ have been employed in each spatial direction. This discretization has been checked by grid convergence tests, carried out for the computation of both the base flow and the unstable eigenvalue. For example, by further increasing the order of the polynomials, the unstable eigenvalue at $R e=240$ (see also the following discussion) changes of about 1\%. For the base flow computation, the simulation is advanced in time at a Reynolds number just below the critical one until a steady state is reached in order to find the base flow $\left(\mathbf{U}_{b}, P_{b}\right)$ for the stability analysis. The linearized/adjoint Navier-Stokes equations are advanced in time with a time step equal to $10^{-3}$, and the solver is used as a time stepper for the stability analysis.

The unstructured FreeFem++ grid employs approximately $3.6 \times 10^{4}$ triangular elements and, again, it is the result of a set of grid convergence tests not shown here for brevity. In this case the unstable eigenvalue at $R e=240$ varies with further grid refinement of less than $2 \%$.

In the plane case, the critical Reynolds number for the primary instability in the mixer is found to be approximately equal to 246 . For validation, a Reynolds number equal to $R e=240$ has been considered for both codes. The resulting eigenvalue associated to the investigated instability has been found equal to $-5.1 \times 10^{-3}$ with NEK5000 and equal to $-5.4 \times 10^{-3}$ with the FreeFem++ codes, the two values being in good agreement. All the partial results of the analysis described in Sec. II, i.e., the direct and adjoint modes as well as the sensitivities with respect to perturbations of the linearized equations and of the base flow, are almost identical when the two different numerical strategies are employed. As an example, we report in Fig. 2 the value of $P_{b}^{+}$on the inflow boundary at $x=-L_{i}$ obtained using NEK5000 and FreeFem ++ , showing that the two results overlap. In agreement with Eq. (7), this term represents practically the sensitivity of the instability with respect to a perturbation of the normal component of the inflow velocity and it collects all the partial results listed in Sec. II, as evident from Eqs. (8). Consequently, Fig. 2 validates at once the results of problems (2), (3), and (8).

\section{RESULTS}

The results shown here have been obtained in the 3D case using a structured multi-block grid. The same 2D grid described in Sec. III is extruded in the $z$ direction, with a uniform distribution of 10 elements. Globally, the grid has approximately 7000 elements, and locally in each element polynomials of order $N=11$ have been employed in each spatial direction, leading to a global 


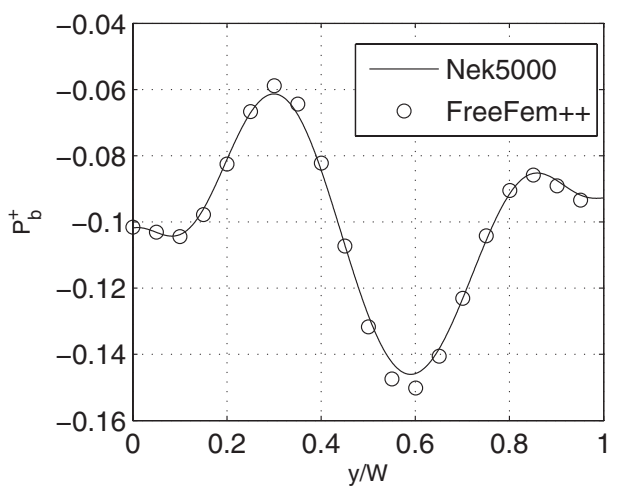

FIG. 2. Adjoint base flow pressure $P_{b}^{+}$obtained with NEK5000 and FreeFem ++ at $R e=240$ at section $x=-L_{i}$ in the plane case.

number of degrees of freedom equal to about $9.4 \times 10^{6}$ for the velocity $\left(5 \times 10^{6}\right.$ for the pressure). The described numerical discretization has been checked by grid convergence tests, not shown here for the sake of brevity, and the resolution in the $x-y$ planes has been chosen on the basis of the results obtained by the validation described in Sec. III. The linearized/adjoint Navier-Stokes equations are advanced in time with a fixed time step equal to $10^{-3}$, and the solver is used as a time stepper for the stability analysis.

\section{A. DNS investigation}

We found that the flow in the T-mixer remains in the vortex regime until $R e=140$. For slightly larger Reynolds numbers the steady engulfment regime is observed. This is in agreement with the numerical results in Galletti et al. ${ }^{6}$ which localize the onset of engulfment in the interval $140<R e<192$ and is exactly the same result as that indicated in Orsi et al..$^{10}$ for the same case. This is also in excellent agreement with Engler $e t a l^{2}(R e=147)$, Bothe $e t a l{ }^{3}(R e=146)$, and Hoffmann $e t$ al. ${ }^{4}$ (Re $\left.=135-150\right)$, who used slightly different geometries.

Let us analyze first the vortex regime, in which the flow is characterized by two counter-rotating vortical structures originating in the recirculation region near the walls of the mixer, at the confluence of the two fluid flows entering, respectively, the two inlet channels (see, e.g., Engler et al., ${ }^{2}$ Bothe et al., ${ }^{3}$ and Hoffmann et al. ${ }^{4}$ ). The vortices are depicted in Fig. 3, where the $\lambda_{2}$-criterion ${ }^{20}$ is used to localize their surface, and the color indicates the sign of the axial vorticity of each vortex, showing that the two vortices are counter-rotating. The vorticity normal to the sections and the on-plane

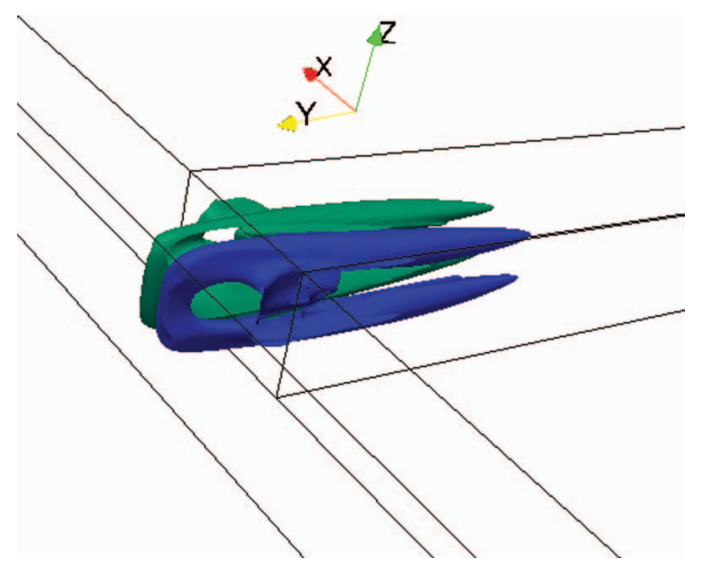

FIG. 3. Vortices identified according to the $\lambda_{2}$-criterion at $R e=140$. 


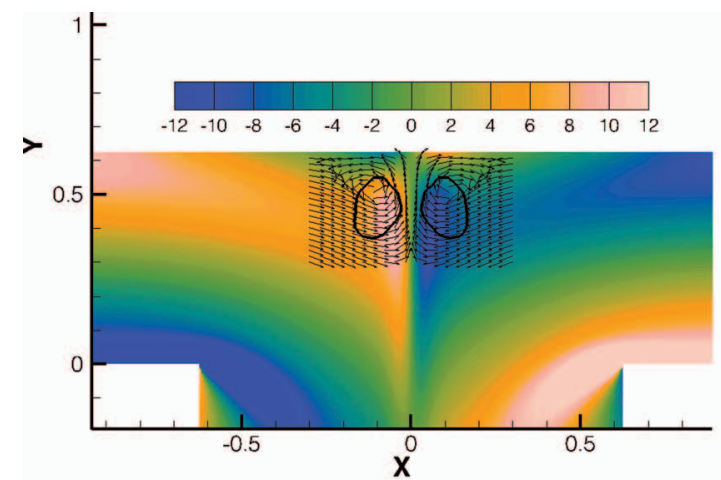

(a)

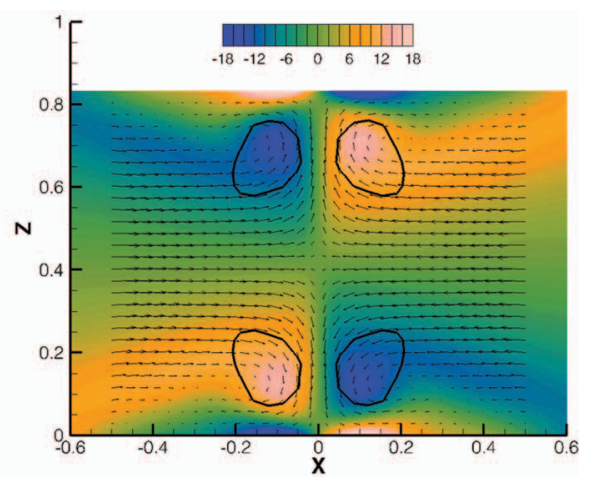

(b)

FIG. 4. $(R e=140)$ Vorticity normal to the cutting plane and in-plane velocity vectors at sections $z=H / 2$ (a) and $y=0.2$ (b); the thick lines indicate the flow region identified as a vortex according to the $\lambda_{2}$ criterion.

velocity vectors are reported for sections $z=H / 2$ and $y=0.2$ in Figs. 4(a) and 4(b), where a thick line indicates the flow region identified as a vortex by the $\lambda_{2}$ criterion. Those figures clearly show that the parts of the two vortices near the top $(y=W)$ and lateral $(z=0, z=H)$ walls of the inlet channels are originated in the separation region forming at the confluence of the two incoming streams. The so-generated vortices are convected by the stream in the outflow pipe, as shown in Fig. 5, where the boundary of the vortices is plotted together with the velocity component normal to the cutting plane at section $y=0.2$, indicating that this velocity component is entirely oriented towards the outflow channel, thus transporting the vortices in that direction. The intensity of the vortices progressively decreases because of diffusion and of interaction with the vorticity generated at the walls. This can be deduced from Fig. 3, looking at the vanishing legs of the vortices in the outflow pipe. Figure 6, reporting the isocontours of the vorticity at two $y$-sections along the outflow channel, highlights the contribution of the new vorticity generated on the walls of the outflow channel to the reduction of the intensity of the vortices. In particular, the vortices loose intensity while progressing towards the outlet channel and they assume a final configuration which is approximately the one depicted in Fig. 6(b). As already evident from the previous figures, the flow and the identified vortices preserve the two reflectional symmetries with respect to planes $z=H / 2$ and $x=0$. Consequently,

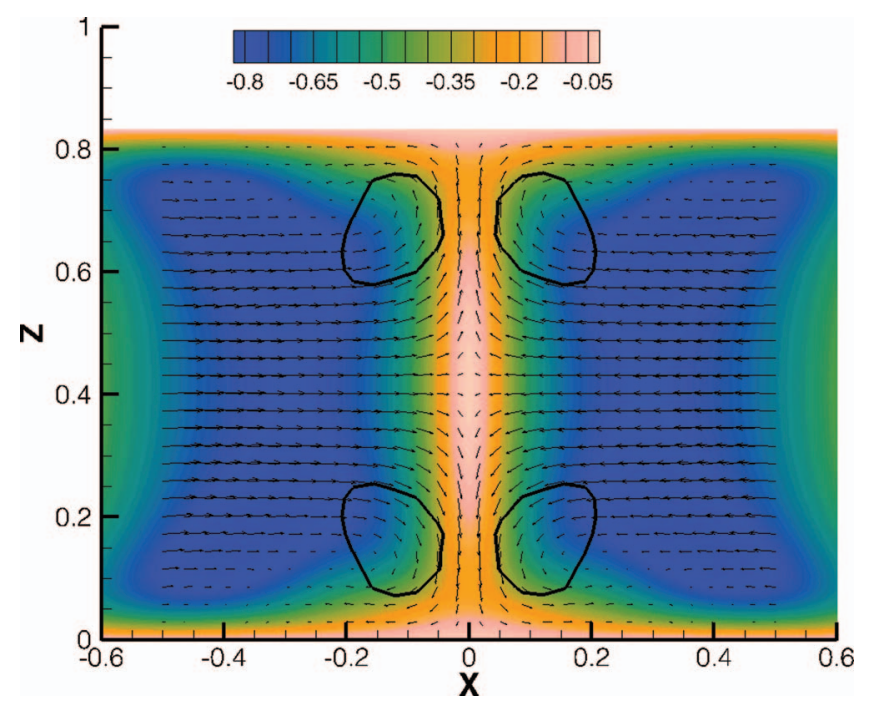

FIG. 5. $(R e=140)$ Velocity in the $y$ direction and in-plane velocity vectors at section $y=0.2$; the thick lines indicate the boundary of the vortices. 


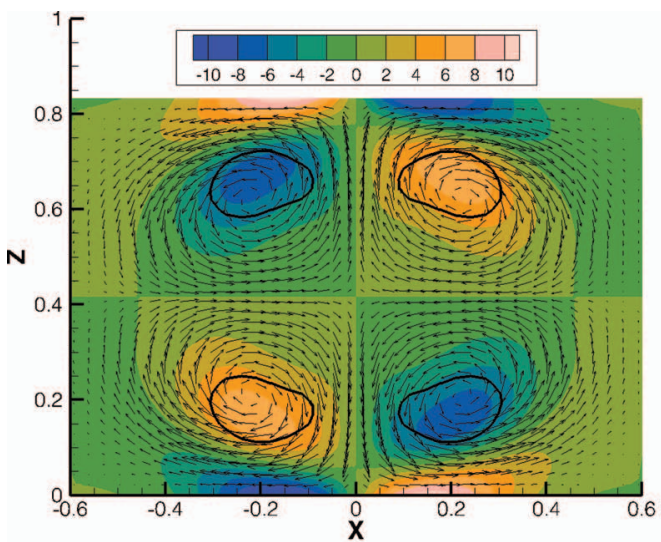

(a)

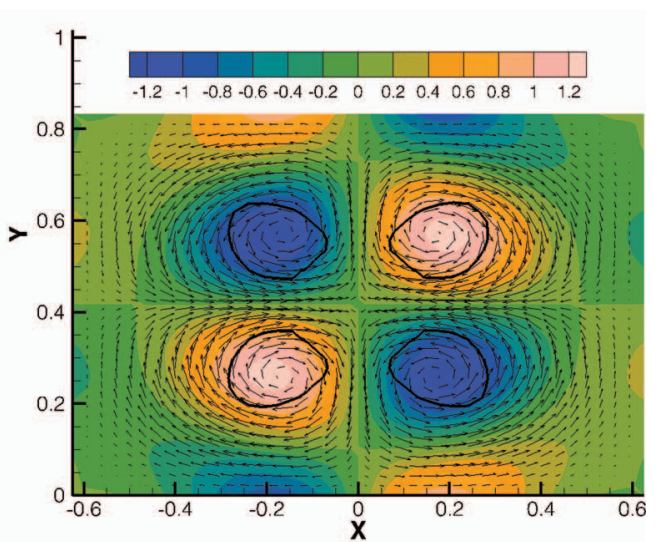

(b)

FIG. 6. $(R e=140)$ Vorticity component in the $y$-direction and in-plane velocity vectors at sections $y=-1$ (a) and $y=-3$ (b); the thick lines indicate the boundary of the vortices.

the two portions of fluid entering from the two inlet channels remain segregated and mixing occurs only through diffusion.

In the engulfment regime the picture changes as follows. First, the reflectional symmetries of the vortex regime are broken, and in particular the two separation bubbles on the top wall of the inlet channels $(y=W)$ tilt in the $x-z$ plane so as to align with one among the two possible diagonal directions of the outlet rectangular section. This is shown in Fig. 7, in which the same quantities as in Fig. 3 are reported but for the engulfment regime. For more details, in Fig. 8 the velocity field (isocontours indicate the normal velocity and vectors the planar components) is shown together with a thick line identifying the vortices for $R e=140$ (a) and $R e=160$ (b). In both cases, the two vortical structures are counter-rotating; consequently the normal velocity induced by these vortices is oriented in the same sense in the region in between of them, as evident in the figure from the high and positive normal velocity region. More interestingly, comparison between Figs. 8(a) and 8(b) highlights the tilting of the recirculation bubbles in the engulfment regime. Moreover, the in-plane velocity vectors indicate that the vortices are also stretched in the $z$-direction.

As a consequence of this new tilted configuration of the separation bubbles on the top wall, each of the two vortices generated in the bubbles is asymmetric, i.e., the two legs of each vortex entering in the outflow channel are not equal in terms of intensity, shape, and position. Moreover, the weakest leg of one vortex is close to the more intense one of the other counter-rotating vortex. Consequently,

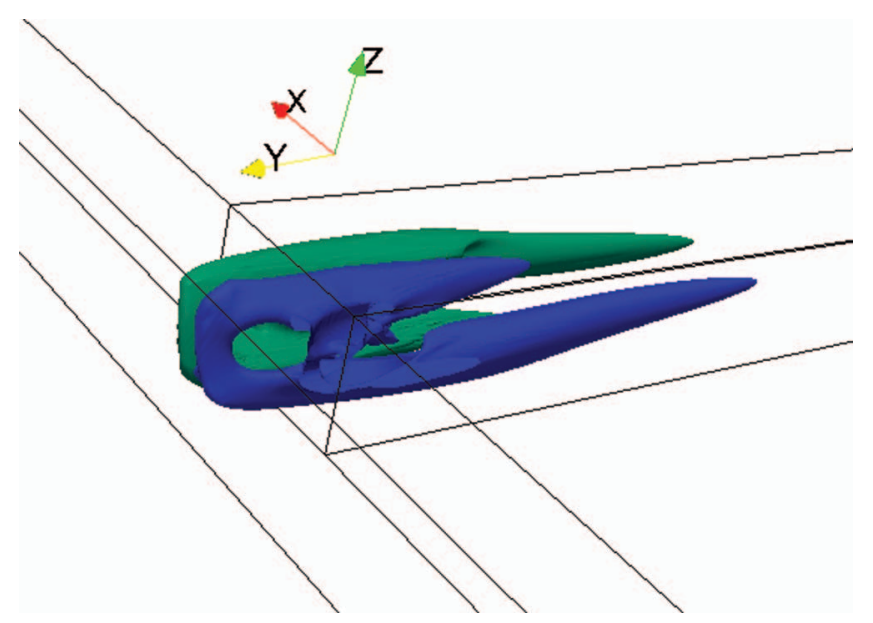

FIG. 7. Vortices identified according to the $\lambda_{2}$-criterion at $R e=160$. 


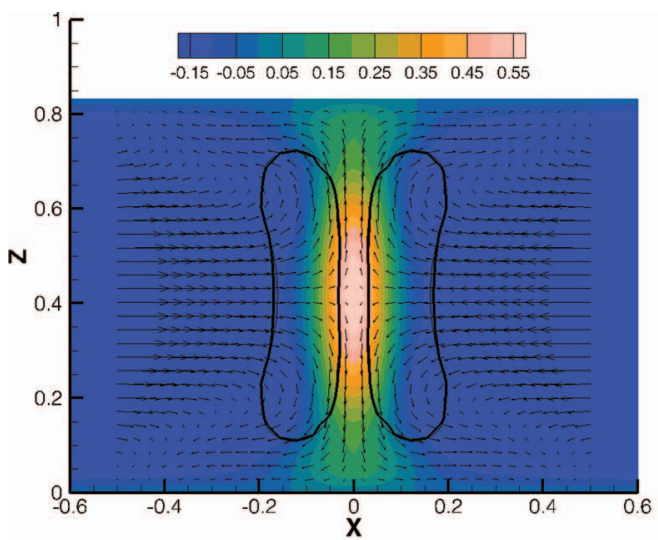

(a)

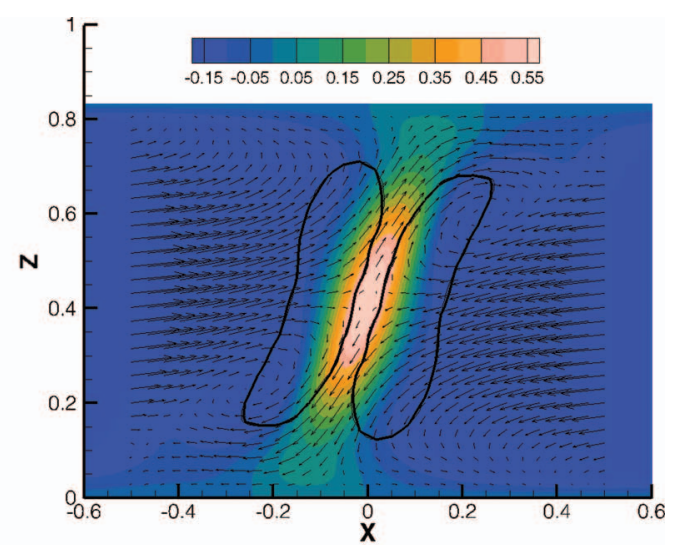

(b)

FIG. 8. Velocity field (iso-contours indicating normal velocity and vectors the planar components) is shown together with a thick line indicating the boundary of the vortices for $R e=140$ (a) and $R e=160$ (b) at section $y=0.4$.

in $x-z$ sections at the beginning of the outflow pipe, four plane vortices can be identified, as shown in Fig. 9(a). Those vortices can be collected in two groups of co-rotating vortices of equal intensity, made, respectively, by the strongest and weakest legs of the previously identified vortical structures. Therefore, the vortices belonging to one of the groups are definitely more intense than the others. Moving towards the end of the outflow channel, the weakest couple of vortices disappears due both to the presence of the other strongest couple of vortices of opposite sign and to the interaction with the walls, as described for the vortex regime. Thus, far enough from the T-junction in the outflow channel, only a couple of co-rotating vortices can be observed, as shown in Fig. 9(b). As also pointed out in the literature, the velocity induced by this couple of co-rotating vortices strongly enhances mixing between the two streams entering the device. This is highlighted here by injecting a passive scalar from one of the two inlet channels. The diffusivity of the scalar has been kept very low, compatibly with the stability restrictions imposed by the numerical scheme $\left(k=10^{-4}, k\right.$ being the diffusivity coefficient of the passive scalar), so as to keep the boundary of the region occupied by the scalar sharp and to highlight the role of convection in the mixing process. Isocontours of the passive scalar are reported together with the vortices in a 3D view in Fig. 10, which clearly shows the role of the velocity induced by the vortical structures in the mixing. This is even more evident in Fig. 11 (section $y=-3$ ) and in Fig. 12 (section $z=H / 2$ ).

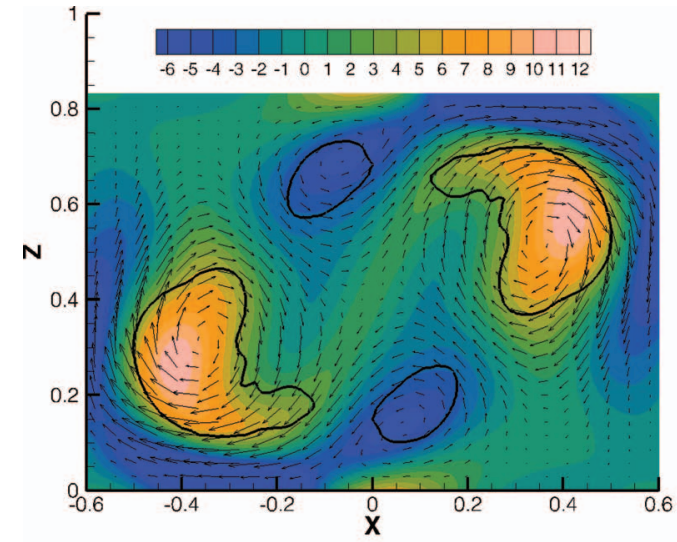

(a)

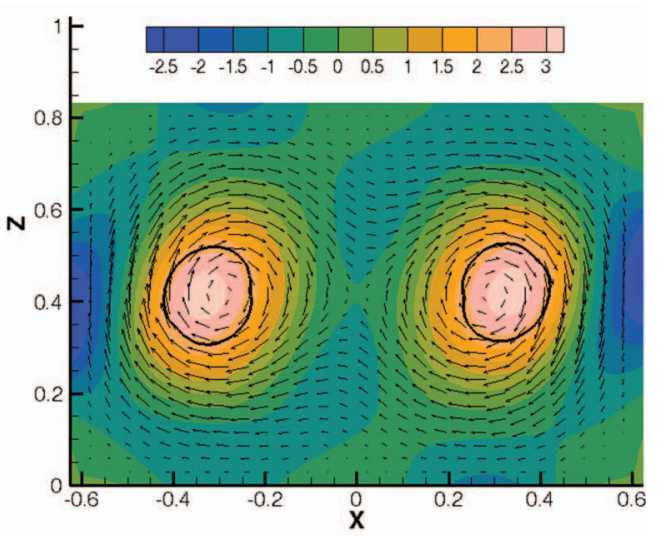

(b)

FIG. 9. $(R e=160)$ Vorticity component in the $y$-direction and in-plane velocity vectors at sections $y=-1$ (a) and $y=-3$ (b); the thick lines indicate the boundary of the vortices. 


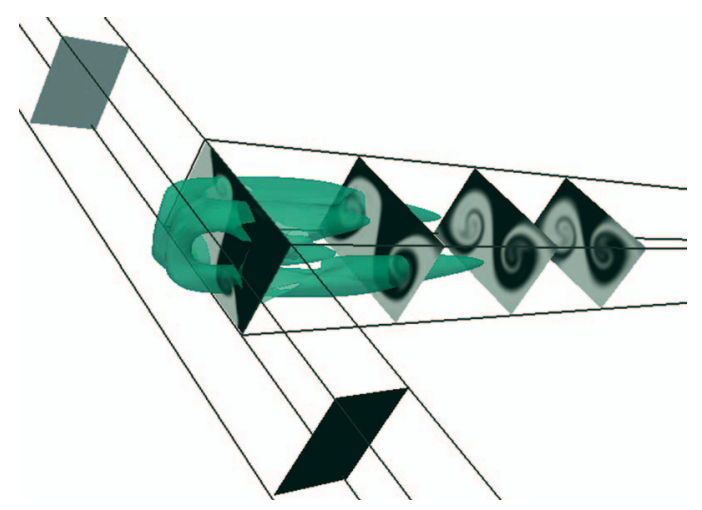

FIG. 10. Vortices identified according to the $\lambda_{2}$-criterion at $R e=160$ and isocontours of the tracer at different $y$-sections along the outflow pipe.

We underline that the figures reported here for the mixing process, and in particular Fig. 12, are in striking agreement with the equivalent experimental and numerical figures reported in Bothe et $a l .{ }^{3}$ for a T-mixer with a slightly different aspect ratio (see, in particular, Fig. 9 of that paper) and with other works available in literature (see, e.g., Engler et $_{\text {al. }}{ }^{2}$ and Hoffmann $e t \mathrm{al}^{4}{ }^{4}$ ). In these previous works the engulfment regime has been characterized mainly in terms of passive scalar concentration and flow pathlines. The description provided here in terms of vortical structures also explains the mechanisms leading to the flow pattern typical of the engulfment regime, which in turn is connected to the significant increase of mixing in this regime. Indeed, from the scenario described above, it is clear that the recirculating regions at the confluence of the two fluids in the inlet channels are critically important for the engulfment instability, since that is the flow region where the two identified vortical structures originate. Moreover, recirculations are fundamental for the onset of global instabilities, since they provide a mean for self-feeding of perturbations. This is confirmed by the localization of the core of the engulfment instability, computed following the method outlined in Sec. II. In particular, the core of the engulfment instability is found to almost coincide with the recirculating regions on the top walls of the inlet channels, thus including the related parts of the two identified vortices. This is shown and commented later in Sec. IV B.

\section{B. Stability and sensitivity analysis}

The solution at $R e=140$ is chosen as base flow for the stability analysis; this Reynolds number is just below the critical one $\left(\sigma=-1.52 \times 10^{-2}\right)$. Since the detected instability is a pitchfork

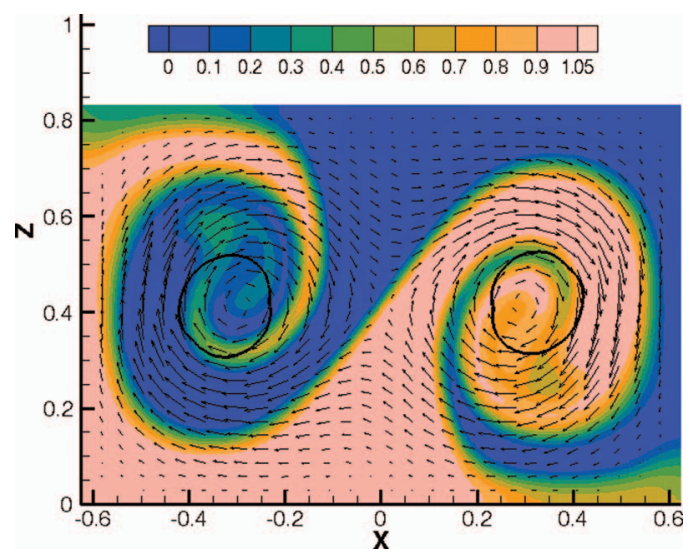

FIG. 11. $(R e=160)$ Passive scalar isocontours and in-plane velocity vectors at plane $y=-3$; the thick lines localize the vortices. 


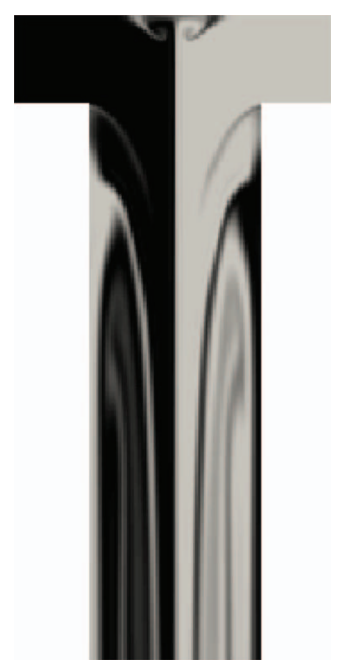

FIG. 12. Passive scalar on plane $z=H / 2$.

bifurcation, the associated eigenvalue and eigenvector are real-valued. The mode is reported in Fig. 13, where the vortical structures associated to it are identified according to the $\lambda_{2}$-criterion; it is clearly shown that the mode is localized in the outflowing pipe and in the region where this intersects the inflow pipes. Selected $y$-sections distributed in the outflow pipe are plotted in Fig. 14. At $y$ sections in the range $y<-3.5$, the mode slowly decays keeping the shape plotted in Fig. 14(d), and it practically vanishes at section $y=-9$. As evident from the figures, the mode is associated to well defined vortical structures, and it is symmetric for reflections with respect to the planes $x=0$ and $z=H / 2$. The shape of the unstable mode is well correlated with the S-shaped engulfment pattern found in the literature ${ }^{2-4,6}$ and with the analysis in Sec. IV A.

In order to study the sensitivity of the instability with respect to the conditions of the incoming flow, we proceed as outlined in Sec. II, and we show in the following the results of the main steps characterizing this analysis. In particular, as a first step, the mode adjoint to the direct one has been evaluated. Unlike the direct mode, the adjoint one is mainly concentrated in the incoming pipes, as reported in Fig. 15 and it is negligibly small in the outflowing one, thus $x$-sections of one of the incoming pipes are shown in Fig. 16, the mode being symmetrical with respect to the plane $x=0$ (i.e., in the other inflow pipe). The mode damps as it approaches the inflow boundaries, while preserving the same shape as that in Fig. 16(c).

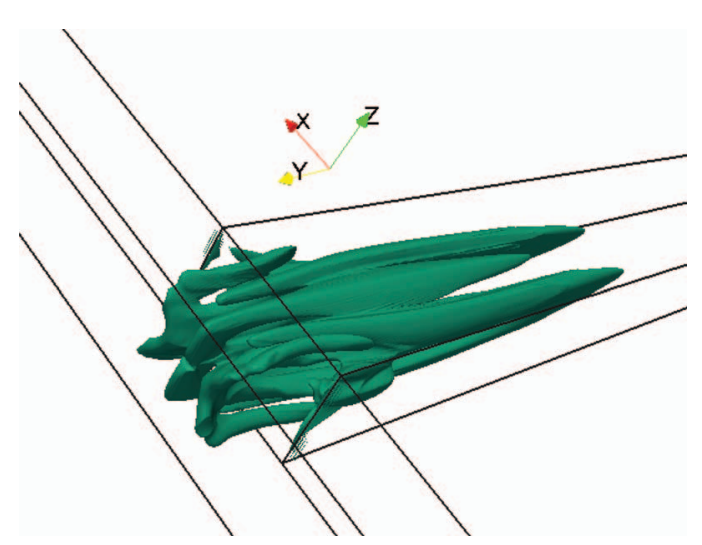

FIG. 13. Vortical structures of the global mode, identified by the $\lambda_{2}$-criterion, computed at $R e=140$. 


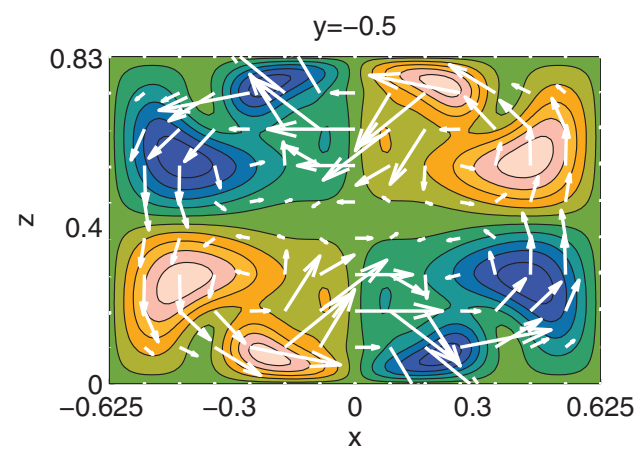

(a)

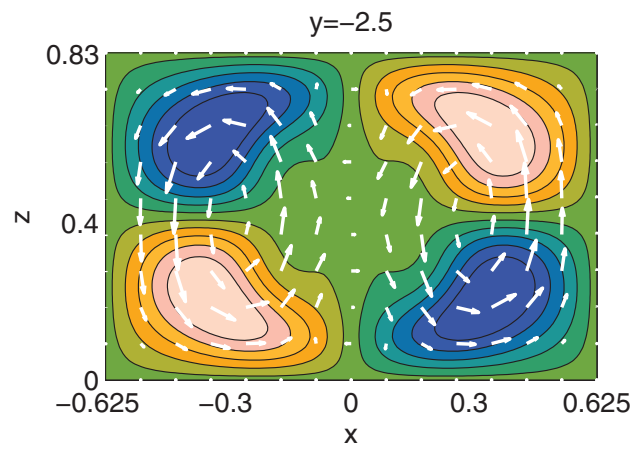

(c)

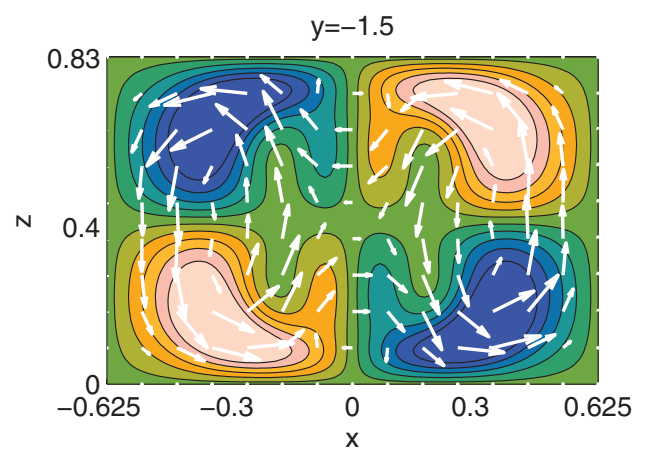

(b)

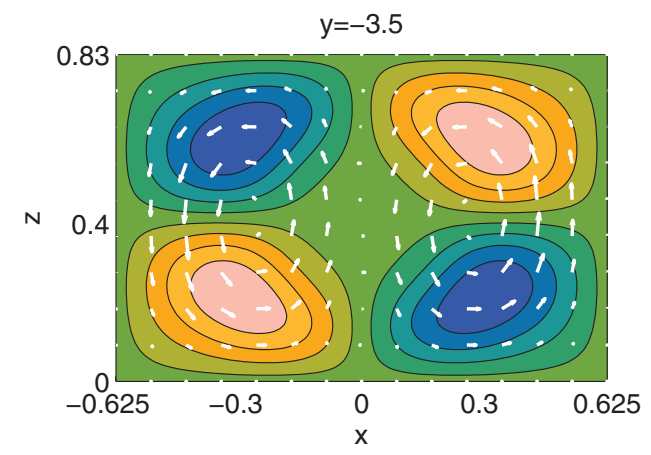

(d)

FIG. 14. (a)-(d) Global direct mode at four different $y$ section: arrows indicate the in-plane velocity components, whose maximum magnitude is approximately equal to 0.72 , and contours represent the velocity component normal to the plane, ranging from -0.6 (dark color) to 0.6 (light color).

The instability core, i.e., the region where instability mechanism acts, is localized in the region where the channels intersect, as shown in Fig. 17(a). Figure 17(b) reports a slice section at $y=0.4$, which shows that field is symmetric with respect to the planes $z=H / 2$ and $x=0$. In particular, the field ||$\hat{\mathbf{u}}|||| \mathbf{u}^{+}||$reaches its maximum inside the recirculation zones where the counterrotating vortices originate. This is again consistent with the analysis reported in Sec. IV A, in which the importance of these recirculation regions in the formation of the vortical structure, which in turn lead to the typical engulfment flow pattern, has been shown.

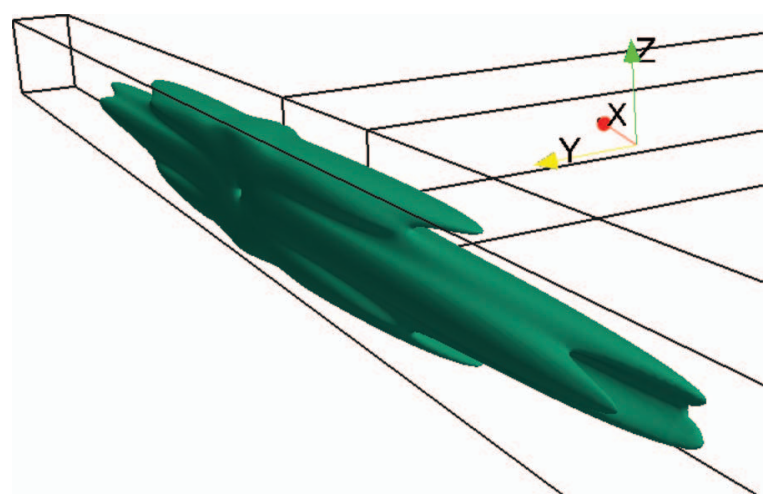

FIG. 15. Isosurface of the magnitude of the adjoint mode velocity $\mathbf{u}^{+}$, computed at $R e=140$. 


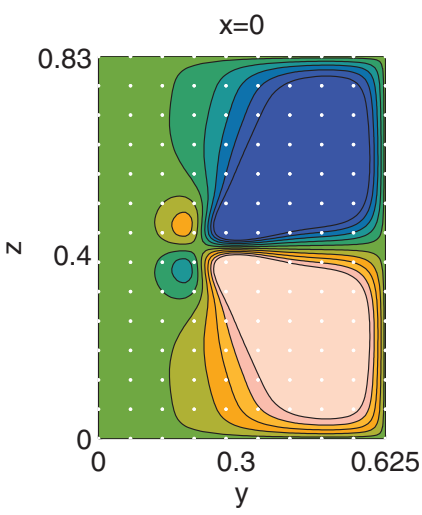

(a)

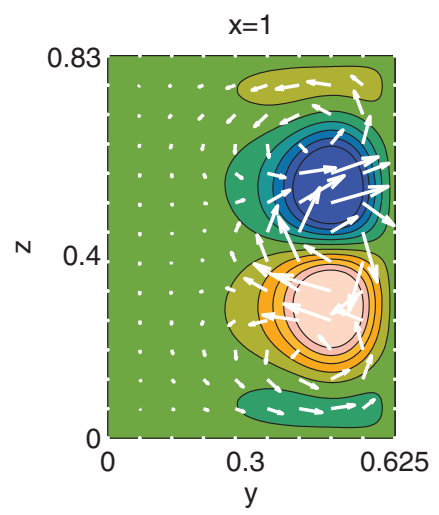

(b)

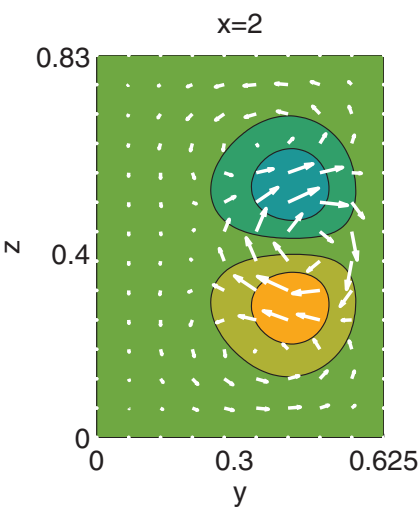

(c)

FIG. 16. (a)-(c) Global adjoint mode at three different $x$ sections: arrows indicate the in-plane velocity components, whose maximum magnitude is approximately equal to 29.2 , and contours represent the velocity component normal to the plane, ranging from -15 (dark color) to 15 (light color).

The adjoint mode is also involved in the computation of the sensitivity of the considered instability with respect to a generic perturbation of the base flow following Eq. (5). This sensitivity, which is represented by the vector field $M^{+}$in Eq. (6) (we remind that normalization (4) is used), is shown in Fig. 18 at three different $x$-sections. The region in which the intensity of $M^{+}$is the largest is the one in which a modification of the base flow would be most effective in promoting or delaying the instability leading to the engulfment. The sensitivity is again localized in the overlapping region between the direct and the adjoint modes, and thus where the three pipes meet. We remark that this becomes practically negligible in the outflow pipe as well as for $\|x\| \geq 1$, and it vanishes in the inflow pipes while preserving the structure depicted in Fig. 18(c).

Let us consider now the sensitivity of the instability to a modification of the incoming velocity profile given by Eq. (7). The sensitivity to an inlet perturbation is given by both the adjoint base flow pressure $P_{b}^{+}$and by a viscous contribution weighted by the inverse of the Reynolds number. The latter term has been found to be negligible, which means that the flow is receptive almost only to a perturbation of the component of the velocity normal to the inflow boundary, since $P_{b}^{+}$acts only in this direction. Therefore, if we consider a localized perturbation of the form $\delta \mathbf{U}_{\mathbf{i}}=U_{i} \delta(y, z) \mathbf{n}$, $\delta(y, z)$ being the 2D Dirac delta function on the inflow plane and $\mathbf{n}$ the normal unit vector to the boundary pointing outside the flow domain, Eq. (7) can be written as $\delta \sigma=U_{i} S(y, z), S$ being a sensitivity map of the eigenvalue with respect to a localized modification of the wall normal component of the inflow velocity. This map is depicted in Fig. 19(a) for section $x=-L_{i}$, the map on the symmetric section $x=L_{i}$ being identical. It is shown that a decrease of the inflow velocity

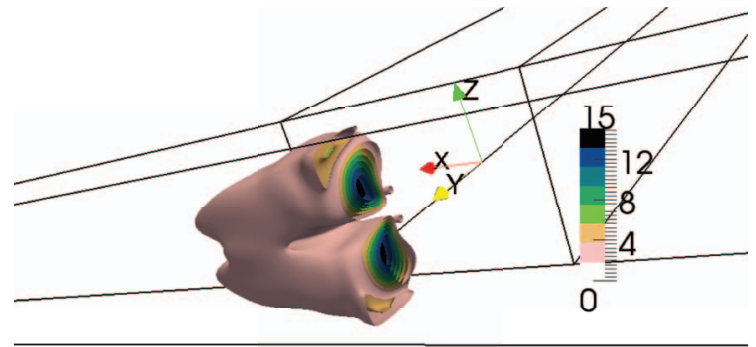

(a)

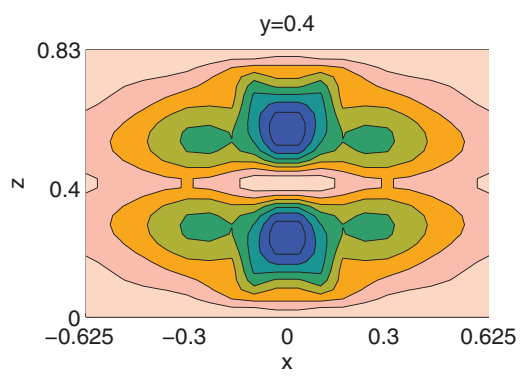

(b)

FIG. 17. Instability core $\|\hat{\mathbf{u}}\|\left\|\mathbf{u}^{+}\right\|$, computed at $R e=140$. (a) 3D view and (b) slice at $y=0.4$. 


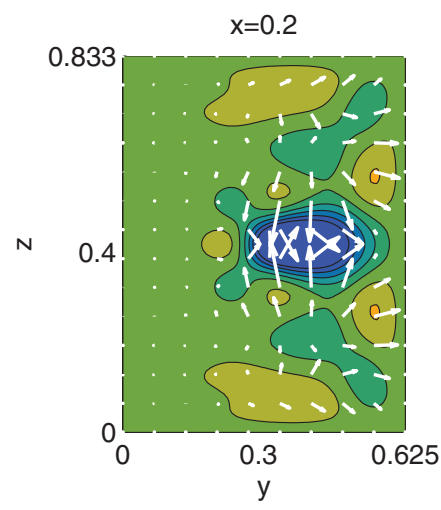

(a)

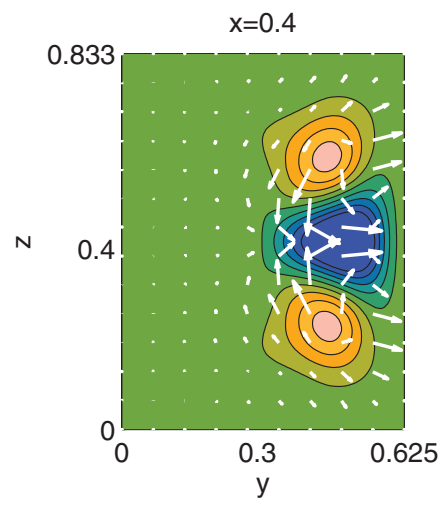

(b)

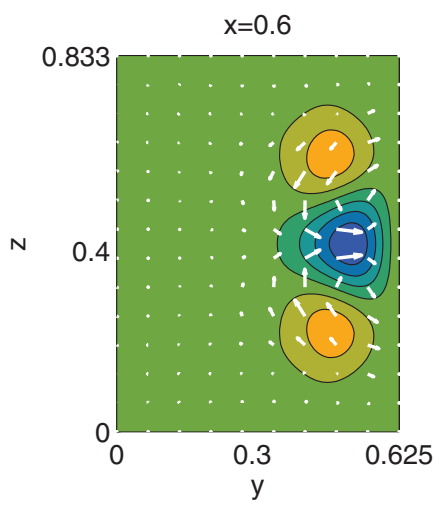

(c)

FIG. 18. Sensitivity to base flow modifications at different $x$ sections: arrows indicate the in-plane sensitivity components, whose maximum magnitude is approximately equal to 66, and contours represent the sensitivity component normal to the plane, ranging from -50 (dark color) to 50 (light color); sections at $x=0.2$ (a), $x=0.4$ (b), and $x=0.6$ (c).

at a generic location of the inflow section, which following the previous definition corresponds to a positive $U_{i}$, always implies a negative shift of the eigenvalue, and this means that the instability eventually leading to engulfment occurs at larger Reynolds numbers. However, Fig. 19(a) also shows that the stabilizing or destabilizing effect depends on the location of the velocity perturbation and that the sensitivity is not symmetric with respect to the plane $y=W / 2$.

As an application and validation of the map provided in Fig. 19(a), we consider a perturbation of the inflow velocity obtained by the following procedure. A DNS simulation (denoted as S2) has been carried out at the same $R e$ (which implies the same mass flux) as the one carried out to compute the base flow in the previous stability and sensitivity analysis, but with a geometry having longer inflow pipes $\left(L_{i}=10\right)$ and with a uniform inflow velocity distribution. In this way, we mimic a case in which the velocity profile is not fully developed at the $x$ sections where sensitivity has been studied ( $\left.x=x_{s i}= \pm 6.875\right)$. We consider as a velocity perturbation $\delta U_{i}$ the difference between the fully developed velocity distribution and the one predicted at $x=x_{s i}= \pm 6.875$ in simulation $\mathrm{S} 2$, reported in Fig. 19(b). It is evident that, as expected, there is a decrease of the inflow velocity in the center of the channel (positive $U_{i}$ ) and an increase in the near wall regions, corresponding to a flatter profile with respect to the fully-developed one, which is typical of non-fully developed conditions (see also Galletti et al. ${ }^{6}$ ). Successively, the unstable eigenvalue has been estimated on the basis of the map in Fig. 19(a), leading to a value $\lambda \simeq-0.010$, which means that the base flow with the non fully-developed velocity profile is slightly more stable than the unperturbed one, which

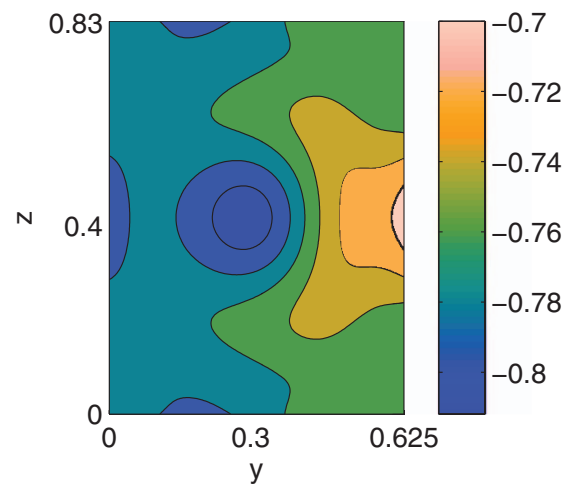

(a)

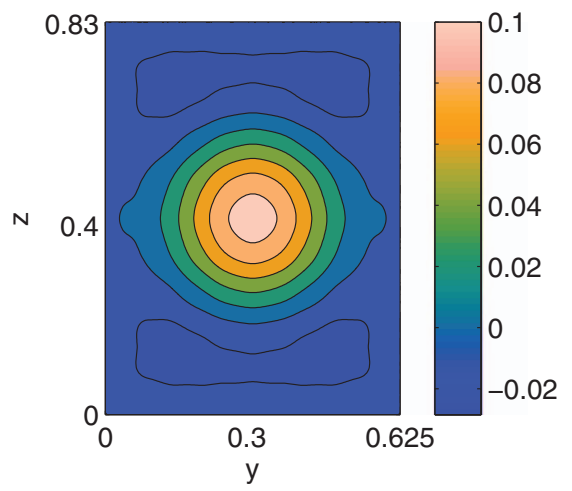

(b)

FIG. 19. Sensitivity to modifications of the velocity profile normal to the inlet plane (a) and considered velocity perturbation (b), both at $x=-L_{i}$. 
was marginally stable at the considered Reynolds number (precisely, with an associated eigenvalue equal to $\lambda_{0} \simeq-2.4 \times 10^{-3}$ ). This is in agreement with the conclusions drawn in Galletti et al. ${ }^{6}$ As a validation, the value of $\lambda$ compares well with the exact one computed by stability analysis carried out on the whole domain of simulation S2, leading to $\lambda_{e x} \simeq-0.014$. We remind that the considered perturbation is not infinitesimal, its maximum intensity being approximately equal to 0.1 , and this is the reason of the discrepancy between $\lambda$ and $\lambda_{e x}$. When the same test was carried out in the 2D case of Sec. III, since the variation of $\lambda$ was one order of magnitude smaller than the present one, the predicted and true values of the eigenvalue of the perturbed problem were in excellent agreement, with discrepancies of the order of $4 \%$. However, a further assessment of the provided 3D maps is detailed in the following. If we consider a velocity perturbation distributed over a surface, e.g., the inflow surface in the previous case, the area-weighted averaged value of the adjoint pressure term in Eq. (7) has no influence only for perturbations $\delta \mathbf{U}_{\mathbf{i}}$ with a global zero mass flux, as the one in Fig. 19(b). Conversely, for perturbations with a net mass flux, the mean value of $P_{b}^{+}$affects the results. However, the problem remains well posed because the outflow boundary condition for the problem (8) involves the value of $P_{b}^{+}$, and consequently its mean value is not arbitrary although only the gradient of $P_{b}^{+}$enters in the field equations. To better illustrate this issue and for a further assessment of the provided sensitivity maps, as a particular case, we consider a perturbation which is proportional to the inflow profile; then the resulting value $\delta \sigma$ is the same as the one that could be found by considering a small variation of the flow Reynolds number, since the bulk velocity is thus changed while keeping the same inflow velocity profile. To this purpose, we consider at $R e=140$ a perturbation intensity such that, when summed to the inflow profile, leads to a bulk velocity equal to $139 / 140$ of the one in the reference case. This configuration is equivalent to one with unit bulk velocity and Reynolds number equal to $R e=139$. Successively, the most unstable eigenmode can be computed directly at $R e=139$ (with unitary bulk velocity) or estimated by the maps derived from the unperturbed case at $R e=140$. By carrying out the linear stability analysis at $R e=139$, the most unstable eigenmode is found to be $\lambda=-7.7 \times 10^{-3}$, while, by using the sensitivity map, this is estimated to be $\lambda=-8.1 \times 10^{-3}$. This test further validates the sensitivity map in Fig. 19(a) and its use in case of perturbations implying a global mass flux variation. Moreover, it confirms that, if the considered perturbation is small enough, the sensitivity maps provide much more accurate predictions.

The same Eq. (7) used here to study the sensitivity to the inlet conditions, can be applied as well to investigate the possibility to control the instability by applying micro-jets on the walls of the mixer. Indeed, Eq. (7) is also valid if the scalar product is obtained by integration not only over $\Gamma_{i}$, as it is written now, but also in general over all the domain boundaries on which Dirichlet boundary conditions are applied for the velocity, as for instance the no-slip walls of the mixer. A variation of the wall-normal component of the velocity on a no-slip wall can be considered as an approximate representation of the effect of a local (suction/blowing) jet, and Eq. (7), integrated on the appropriate boundaries, can thus characterize the effects of such a control on the instability. An example is given in Fig. 20, where the sensitivity $S$ of the instability is characterized with respect to the introduction of a localized velocity, normal to the boundary, on the walls $y=W$ and $z=0$ (in the latter case only the more interesting part of the wall surface is shown). In particular, a jet implies a negative wall-normal velocity with respect to the normal pointing outside the flow domain, thus it can have

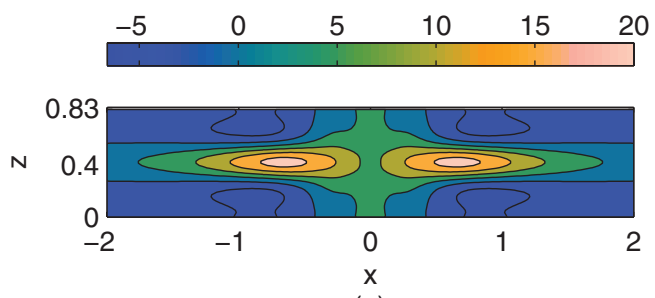

(a)

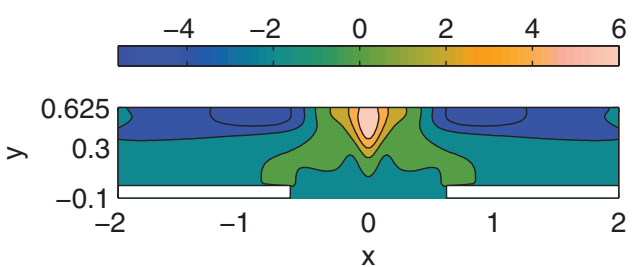

(b)

FIG. 20. Sensitivity to introduction of a velocity profile normal to wall; sections at $y=W$ (a) and at $z=0$ (b). 
a stabilizing effect (i.e., $\delta \lambda<0$ ) if placed in regions of the maps in Fig. 20 where the value of sensitivity is positive (see Eq. (7) replacing $\Gamma_{i}$ with the appropriate boundary face). Conversely, a hole with suction is stabilizing if placed in regions where $S$ is negative valued. Thus, maps in Fig. 20 provides a guideline for an active control of the instability through suction/blowing from the walls, quantifying the effect of a local introduction of such a device as a function of the position where this is introduced.

\section{CONCLUSIONS}

Direct numerical simulation and three-dimensional linear instability and sensitivity analyses, involving the computation of the direct and adjoint global modes, have been applied to the investigation of the onset of the steady engulfment regime in a micro T-mixer. The computations for stability and sensitivity analysis of fully 3D configurations without any homogeneous direction imply a significant technical complexity and large computational costs. Ad hoc numerical tools have been developed using the spectral-element code NEK5000; their validation has been presented herein.

First, direct numerical simulations have shown that the key mechanism for engulfment onset is the tilting of the vortical structures originating at the confluence of the two streams in the inlet channels, which leads to the break of the symmetry of the two pairs of counter-rotating vortices entering the mixing channel. As a consequence, one of the vortices of each pair becomes more intense than the other; the weaker one progressively disappears moving downstream in the mixing channel. Therefore, the largest part of the mixing channel is characterized by the presence of only two co-rotating vortical structures, typical of the engulfment regime, and yielding to the enhancement of mixing.

Stability and sensitivity analyses have then been carried out. Fully-developed inlet velocity conditions have been first considered as in the direct numerical simulations. The critical Reynolds number computed by stability analysis for this case is in good agreement with the findings of our direct numerical simulations and with those in the literature. The shape of the unstable mode given by the linear stability analysis is very well correlated with the flow and vorticity patterns observed in DNS. Moreover, the core of the instability, i.e., the overlapping region between the direct and adjoint unstable modes, has been found to coincide with the recirculation regions where the identified vortical structures originate, in agreement with the scenario proposed from DNS results.

The information given by the direct and adjoint fields has also been used to compute the sensitivity of the instability with respect to a perturbation the velocity distribution at the inlet of the T-mixer. As a particular application, a perturbation of the inlet velocity has been considered such that, once added to the fully-developed inlet velocity, leads to inlet conditions typical of a non-fully developed case. The sensitivity analysis shows that for the non-fully developed case the flow tends to be more stable. This is in agreement with the instability analysis directly applied to the non-fully developed case and with the indications given in the literature. Finally, the same sensitivity analysis is used to investigate the possibility to control the instability by applying micro-jets on the walls of the mixer. The so obtained sensitivity maps provide a guideline for an active control of the instability through suction/blowing from the walls, quantifying the effect of a local introduction of such a device as a function of its location.

The presented numerical framework can be straightforwardly applied to different T-mixer geometries and to the design and appraisal of various control strategies.

\section{ACKNOWLEDGMENTS}

CASPUR (Rome, Italy) and CINECA (Bologna, Italy) computing centers are gratefully acknowledged for allowance of computational resources. The authors wish to thank E. Brunazzi, C. Galletti, and R. Mauri for having stimulated this study.

\footnotetext{
${ }^{1}$ V. Kumar, M. Paraschivoiu, and K. D. P. Nigam, "Single-phase fluid flow and mixing in microchannels," Chem. Eng. Sci. 66, 1329-1373 (2011).

${ }^{2}$ M. Engler, N. Kockmann, T. Kiefer, and P. Woias, "Numerical and experimental investigations on liquid mixing in static micromixers," Chem. Eng. J. 101, 315-322 (2004).
} 
${ }^{3}$ D. Bothe, C. Stemich, and H. J. Warnecke, "Fluid mixing in a T-shaped micro-mixer," Chem. Eng. Sci. 61, 2950-2958 (2006).

${ }^{4}$ M. Hoffmann, M. Schlüter, and N. Räbiger, "Experimental investigation of liquid-liquid mixing in T-shaped micro-mixers using $\mu$-LIF and $\mu$-PIV," Chem. Eng. Sci. 61, 2968-2976 (2006).

${ }^{5}$ S. Dreher, N. Kockmann, and P. Woias, "Characterization of laminar transient flow regimes and mixing in T-shaped micromixers," Heat Transfer Eng. 30, 91-100 (2009).

${ }^{6}$ C. Galletti, M. Roudgar, E. Brunazzi, and R. Mauri, "Effect of inlet conditions on the engulfment pattern in a T-shaped micro-mixer," Chem. Eng. J. 185, 300-313 (2012).

${ }^{7}$ A. Soleymani, H. Yousefi, and I. Turunen, "Dimensionless number for identification of flow patterns inside a T-micromixer," Chem. Eng. Sci. 63, 5291-5297 (2008).

${ }^{8}$ S. K. R. Cherlo and S. Pushpavanam, "Effect of depth on onset of engulfment in rectangular micro-channels," Chem. Eng. Sci. 65, 6486-6490 (2010).

${ }^{9}$ H. Yousefi, W. Ratchananusorn, I. Turunen, and A. Soleymani, “Pressure drop in micro T-mixers," J. Micromech. Microeng. 20, 15029-15035 (2010).

${ }^{10}$ G. Orsi, M. Roudgar, E. Brunazzi, C. Galletti, and R. Mauri, "Water-ethanol mixing in T-shaped microdevices," Chem. Eng. Sci. 95, 174-183 (2013).

${ }^{11}$ V. Theofilis, "Global linear instability," Annu. Rev. Fluid Mech. 43, 319-352 (2011).

${ }^{12}$ A. Bottaro, P. Corbett, and P. Luchini, "The effect of base flow variation on flow stability," J. Fluid Mech. 476, 293-302 (2003).

${ }^{13}$ F. Giannetti and P. Luchini, "Structural sensitivity of the first instability of the cylinder wake," J. Fluid Mech. 581, 167-197 (2007).

${ }^{14}$ O. Marquet, D. Sipp, and L. Jacquin, "Sensitivity analysis and passive control of cylinder flow," J. Fluid Mech. 615, 221-252 (2008).

${ }^{15}$ S. Bagheri, P. Schlatter, P. J. Schmid, and D. S. Henningson, "Global stability of a jet in crossflow," J. Fluid Mech. 624, 33-44 (2009).

${ }^{16}$ P. F. Fischer, J. W. Lottes, and S. G. Kerkemeier, “NEK5000 web page,” 2008, see http://nek5000.mcs.anl.gov.

${ }^{17}$ A. Fani, S. Camarri, and M. V. Salvetti, "Stability analysis and control of the flow in a symmetric channel with a sudden expansion," Phys. Fluids 24, 084102 (2012).

${ }^{18}$ P. Meliga and J. M. Chomaz, "Global modes in a confined impinging jet: application to heat transfer and control," Theor. Comput. Fluid Dyn. 25, 179-193 (2011).

${ }^{19}$ S. Balay, J. Brown, K. Buschelman, V. Eijkhout, W. D. Gropp, D. Kaushik, M. G. Knepley, L. C. McInnes, B. F. Smith, and H. Zhang, "PETSc users manual," Technical Report No. ANL-95/11 - Revision 3.4 (Argonne National Laboratory, 2013).

${ }^{20}$ J. Jeong and F. Hussain, "On the identification of a vortex,” J. Fluid Mech. 285, 69-94 (1995). 